\title{
Slowly Progressive Clinical Course
}

National Cancer Institute

\section{Source}

National Cancer Institute. Slowly Progressive Clinical Course. NCI Thesaurus. Code C115461.

Clinical course characterized by slow progression without signs of aggressive disease. 